International Mathematical Forum, 2, 2007, no. 29, 1445 - 1456

\title{
Some Aging Classes of Life Distributions at Specific Age
}

\author{
I. Elbatal \\ King Saud University \\ College of Science, Dept. of Stat. and O. R, \\ P.O. Box 2455 Riyadh 11451, Saudi Arabia \\ i_elbatal@yahoo.com
}

\begin{abstract}
We introduce some properties of the new better than used in convex ordering at age $\mathrm{t}_{0}\left(N B U C-t_{0}\right)$ and new better than used of second order $(2)$ at age $t_{0}\left(N B U(2)-t_{0}\right)$ classes of life distributions, where the survival probability at age 0 is greater than or equal to the conditional survival probability at specified age $t_{0}>0$. Preservation properties of the two classes under various reliability operations and shock model are arriving according to homogeneous Poisson process are established.
\end{abstract}

Keywords: $N B U C-t_{0} ; N B U(2)-t_{0}$; preservation properties; shock model; parallel system

\section{Introduction}

The notion of aging for engineering systems has been characterized by several classes of life distributions in reliability various classes of life distributions have been introduced to describe several types of deterioration (improvement) that accompany aging. In performing reliability analysis, it has been found very useful to classify life distributions using the concept of stochastic ordering. For definitions of several classes of distributions,e.g., IF R, IFRA, NBU, $N B U E, N B U C$ and $N B U(2)$. For more detailed discussions on properties and for some possible applications, we refer to Bryson and Siddiqui (1969), Rolski (1975), Barlow and Proschan (1981), Deshpand et al (1986) and Cao and Wang (1991). Unfortunately these types of classes are restricted to one type of applications since they present positive aging or negative aging for their dual classes throughout their life span of the underlying components or their systems. Hollander et al. (1986) have introduced the new better than used at specific age at $t_{0}\left(N B U-t_{0}\right)$ and its dual $\left(N W U-t_{0}\right)$. They have listed some types of situation where $\left(N B U-t_{0}\right)$ aging or its dual might arise. 
There are many situation in real life where the components of the system gradually deteriorate up time $t_{0}$ which is warranty guarantee time provided by most manufacturers, then maintenance through repairs or spare parts replacement take place after tomet $t_{0}$. Here maintenance is expected to improve the performance of the system but can not bring it back to a better situation than it was at age $t_{0}$. For some interesting examples, see Hollander at al.(1986).

In this paper we further develop some of the above mentioned classes. In Section 2 we will recall some definitions and facts about characteristics of lifetime distributions and about stochastic ordering. In Section 3 we discuss whether the $N B U C-t_{0}$ and $N B U(2)-t_{0}$ are preserved under convolution. In Section 4, we show that $N B U C-t_{0}$ class is closed under the formation of parallel sytems. Finally, $N B U C-t_{0}$ under shock model is discussed in Section 5.

\section{Definitions and relationships}

In reliability theory, aging life is usually characterized by a nonnegative random variable $X \geq 0$ with cumulative distribution function (cdf) $F$ and survival function (sf) $\bar{F}=1-F$. For any random variable $X$, let

$$
X_{t}=[X-t \mid X>t], \quad t \in\{x: F(x)<1\},
$$

denote a random variable whose distribution is the same as the conditional distribution of $X-t$ given that $X>t$. When $X$ is the lifetime of a device, $X_{t}$ can be regarded as the residual lifetime of the device at time $t$, given that the device has survived up to time $t$. Its survival function is (see, for instance, Deshpand et al. (1986))

$$
\overline{F_{t}}(x)=\frac{\bar{F}(t+x)}{\bar{F}(t)}, \quad \bar{F}(t)>0,
$$

where $\bar{F}(x)$ is the survival function of $X$. It is well-known fact that when $\bar{F}$ is an exponential distribution then $X_{t} \stackrel{s t}{=} X$ or $\overline{F_{t}}(x)=\bar{F}(x)$. Comparing $X$ and $X_{t}$ in various forms and types create classes of aging useful in many biomedical, engineering and statistical studies, cf. Barlow and Proschan (1981). It is wellknown that the relation $X_{t} \stackrel{s t}{\leq} X$ or $\overline{F_{t}}(x) \stackrel{s t}{\leq} \bar{F}(x)$ defines the class of new better than used $(N B U)$.

\section{Definition 2.1.}

A non-negative random variable $X$, or it is distribution $F$, is said to be:

(i) new better than used (denoted by $X \in N B U$ ) if

$$
\bar{F}(t+y) \leq \bar{F}(t) \bar{F}(y), \quad \text { for all } t \geq 0 ;
$$


(ii) new better than used in the convex order (denoted by $X \in N B U C$ ) if

$$
\int_{x}^{\infty} \bar{F}(t+y) d y \leq \bar{F}(t) \int_{x}^{\infty} \bar{F}(y) d y \quad \text { for all } \quad x, \quad t \geq 0 .
$$

(iii) new better than used of second $\operatorname{order}(2)$ (denoted by $X \in N B U(2)$ ) if

$$
\int_{0}^{y} \bar{F}(t+x) d x \leq \bar{F}(t) \int_{0}^{y} \bar{F}(x) d x
$$

We have the following chain of implications of the above mentioned classes as follows:

$$
N B U C \Leftarrow N B U \Rightarrow N B U(2)
$$

Hollander et al (1986) introdused the larger class of life distributions called new better than used of age $t_{0}$ defined as follows: A non-negative random variable $X$ is said to be new better than used of age $t_{0}$ if

$$
\bar{F}\left(t_{0}+x\right) \leq \bar{F}\left(t_{0}\right) \bar{F}(x), \quad \text { for all } x \geq 0 .
$$

Thus the $N B U$ property states that a used item of any age has stochastically smaller residual life length than does a new item, whereas the $N B U-t_{0}$ property states that a used item of age $t_{0}$ has stochastically smaller residual life length than does a new item.

A ccording to the above definition we introduce the concept of new better than used in convex ordering and new better than used of second order (2) classes of age $t_{0}$ as follows.

\section{Definition 2.2.}

A life distribution $F$ is said to be

(i) new better than used in convex ordering of age $t_{0}\left(N B U C-t_{0}\right)$ if

$$
\int_{x}^{\infty} \bar{F}\left(y+t_{0}\right) d y \leq \bar{F}\left(t_{0}\right) \int_{x}^{\infty} \bar{F}(y) d y \text { for all } x \geq 0, t_{0}>0 .
$$

(ii) new better than used of second order(2)of age $t_{0}\left(N B U(2)-t_{0}\right)$ if

$$
\int_{0}^{x} \bar{F}\left(y+t_{0}\right) d y \leq \bar{F}\left(t_{0}\right) \int_{0}^{x} \bar{F}(y) d y, \text { for all } x \geq 0 .
$$

Remarke: It is seen from the above results that the $N B U(2)-t_{0}$ class is a middle class between the $N B U-t_{0}$ and $N B U E-t_{0}$ classes. This 
of course would prove useful in applications since it is less restrictive than $N B U-t_{0}$ and easier to verify in practice than $N B U E-t_{0}$. Note that the $N B U-t_{0}$ notion of aging compares a new unit with used units of all possible age in specific age $t_{0}$. On might look at the $N B U(2)-t_{0}$ property as comparing the average performance of corresponding used units in specific age $t_{0}$ which is more appealing in practice.

\section{Closure properties}

As an important reliability operation,convolution of life distributions of certain class is often paid much attention.It has been shown that both the $N B U C-t_{0}$ and $N B U(2)-t_{0}$ classes are closed under this operation.In the next theorems we established the clouser property of the $N B U C-t_{0}$ and $N B U(2)-t_{0}$ under the convolution operation.

Theorem(3.1).Suppose that $F_{1}$ and $F_{2}$ are two independent $N B U C-t_{0}$ life distributions. Then their convolution is also

$N B U C-t_{0}$.

Proof: The survival function of the convolution of two life distributions $F_{1}$ and $F_{2}$ is

$$
\bar{F}(z)=\int_{0}^{\infty} \bar{F}_{1}(z-u) d F_{2}(u) d u \quad \text { for all } z \geq 0 .
$$

For any fixed $x \geq 0$

$$
\begin{gathered}
\int_{x}^{\infty} \bar{F}\left(y+t_{0}\right) d y=\int_{0}^{\infty} \int_{0}^{\infty} \overline{F_{1}}\left(x+t_{0}+y-u\right) d F_{2}(u) d y \\
=\int_{0}^{\infty} \int_{0}^{x} \overline{F_{1}}\left(x+t_{0}+y-u\right) d F_{2}(u) d y+\int_{0}^{\infty} \int_{x}^{\infty} \overline{F_{1}}\left(x+t_{0}+y-u\right) d F_{2}(u) d y \\
=\int_{0}^{x}\left[\int_{0}^{\infty} \overline{F_{1}}\left(x+t_{0}+y-u\right) d y\right] d F_{2}(u) \\
+\int_{0}^{\infty}\left[\int_{0}^{\infty} \overline{F_{1}}\left(t_{0}+y-w\right) d_{w} F_{2}(x+w] d y\right. \\
=A_{1}+A_{2}
\end{gathered}
$$

Not that $\bar{F}\left(t_{0}\right) \geq \bar{F}_{i}\left(t_{0}\right) \quad$ and $\quad i=1,2$. It follows from the $N B U C-t_{0}$ property of $F_{1}$ that 


$$
\begin{aligned}
A_{1} & \leq \overline{F_{1}}\left(t_{0}\right) \int_{0}^{x}\left[\int_{0}^{\infty} \overline{F_{1}}(x+y-u) d y F_{2}(u)\right] d F_{2}(u) \\
& \leq \bar{F}\left(t_{0}\right) \int_{0}^{\infty} \int_{0}^{x} \overline{F_{1}}(x+y-u) d F_{2}(u) d y
\end{aligned}
$$

Integrating by parts in the inner integral of $A_{2}$ we have

$$
\begin{gathered}
A_{2}=\int_{0}^{\infty}\left[\overline{F_{1}}\left(t_{0}+y\right) \overline{F_{2}}(x)+\int_{0}^{\infty} \overline{F_{2}}(x+w) d_{w} \overline{F_{1}}\left(y+t_{0}-w\right)\right] d y \\
=\overline{F_{2}}(x) \int_{0}^{\infty} \overline{F_{1}}\left(t_{0}+y\right) d y+\int_{0}^{\infty}\left[\int_{0}^{y+t_{0}} \overline{F_{2}}(x+w) d_{w} \overline{F_{1}}\left(y+t_{0}-w\right)\right] d y \\
=\phi_{1}+\int_{0}^{\infty}\left[\int_{0}^{y+t_{0}} \overline{F_{2}}\left(x+t_{0}+y-u\right) d F_{1}(u)\right] d y \\
=\phi_{1}+\int_{t_{0}}^{\infty}\left[\int_{u-t_{0}}^{\infty} \overline{F_{2}}\left(x+t_{0}+y-u\right) d y\right] d F_{1}(u) \\
+\int_{0}^{t_{0}}\left[\int_{0}^{\infty} \overline{F_{2}}\left(x+t_{0}+y-u\right) d y\right] d F_{1}(u) \\
=\phi_{1}+\phi_{2}+\phi_{3}
\end{gathered}
$$

where

$$
\begin{gathered}
\phi_{1} \leq \mu_{1} \overline{F_{2}}(x) \overline{F_{1}}\left(t_{0}\right) \leq \mu_{1} \overline{F_{2}}(x) \bar{F}\left(t_{0}\right) \\
\phi_{2}=\overline{F_{1}}\left(t_{0}\right) \int_{0}^{\infty} \overline{F_{2}}(x+u) d u \\
\phi_{3} \leq \int_{0}^{t_{0}}\left[\overline{F_{2}}\left(t_{0}-u\right) \int_{0}^{\infty} \overline{F_{2}}(x+y) d y\right] d F_{1}(u) \\
=\int_{0}^{\infty} \overline{F_{2}}(x+y) d y \int_{0}^{t_{0}} \overline{F_{2}}\left(t_{0}-u\right) d F_{1}(u) \\
=\left[\bar{F}\left(t_{0}\right)-\overline{F_{1}}\left(t_{0}\right)\right] \int_{0}^{\infty} \overline{F_{2}}(x+u) d u .
\end{gathered}
$$

by the $N B U C-t_{0}$ property of $F_{1}, F_{2}$ and $\mu_{1}=\int_{0}^{\infty} \overline{F_{1}}(u) d u$.then from $(3.1)-(3.4)$ 
we get

$$
A_{2} \leq \bar{F}\left(t_{0}\right)\left[\mu_{1} \overline{F_{2}}(x)+\int_{0}^{\infty} \overline{F_{2}}(x+u) d u\right] .
$$

On the other hand

$$
\begin{gathered}
\bar{F}\left(t_{0}\right) \int_{x}^{\infty} \bar{F}(y) d y \\
=\bar{F}\left(t_{0}\right)\left[\int_{0}^{\infty} \int_{0}^{x} \overline{F_{1}}(x+y-u) d F_{2}(u) d y+\int_{0}^{\infty} \overline{F_{2}}(x+u) d u+\mu_{1} \overline{F_{2}}(x)\right] \\
\geq A_{1}+A_{2}=\int_{x}^{\infty} \bar{F}\left(y+t_{0}\right) d y .
\end{gathered}
$$

This proves that $F$ is $N B U C-t_{0}$.

Theorem(3.2). Suppose that $F_{1}$ and $F_{2}$ are two independent $N B U(2)-t_{0}$ life distributions. Then their convolution is also $N B U(2)-t_{0}$.

Proof: The survival function of the convolution of two life distributions $F_{1}$ and $F_{2}$ is

$$
\bar{F}(y)=\int_{0}^{\infty} \bar{F}_{1}(y-u) d F_{2}(u) d u
$$

By Fubini's theorem and the $N B U(2)-t_{0}$ property, we have

$$
\begin{gathered}
\int_{0}^{x} \bar{F}\left(y+t_{0}\right) d y=\int_{0}^{x} \int_{0}^{\infty} \bar{F}\left(y+t_{0}-u\right) d F_{2}(u) d y \\
=\int_{0}^{\infty} \int_{0}^{x} \overline{F_{1}}\left(y+t_{0}-u\right) d y d F_{2}(u) \\
\leq \int_{0}^{\infty} \overline{F_{1}}\left(t_{0}\right) \int_{0}^{x} \overline{F_{1}}(y-u) d y d F_{2}(u) \\
=\overline{F_{1}}\left(t_{0}\right) \int_{0}^{x} \int_{0}^{\infty} \overline{F_{1}}(y-u) d F_{2}(u) d y \\
\leq \bar{F}\left(t_{0}\right) \int_{0}^{x} \overline{F_{1}}(y) d y,
\end{gathered}
$$

the last inequality following from the fact that the convolution of two independent nonnegative random variables is stochastically larger than each of them. This proves that $F$ is also $N B U(2)-t_{0}$. 


\section{Closure of the NBUC- $t_{0}$ class under for- mation of parallel system.}

Hendi et al (1993) have shown that the NBUC class is closed under formation of parallel system of i.i.d.components. Here it may be of interest to prove the closure of the $N B U C-t_{0}$ class under formation of parallel systems in the following theorem.

Theorem(4.1).Let $X_{1}, X_{2}, \ldots, X_{n}$ be i.i.d. random variables with distribution $F$ and $F \in N B U C-t_{0}$. Then the random variable $X_{n}=\max \left(X_{1}\right.$ $\left., X_{2}, \ldots, X_{n}\right)$ has distribution $F_{n} \in N B U C-t_{0}$.

Proof:

The survival function of $X_{n}$ is $\overline{F_{(n)}}\left(t_{0}\right)=P\left(X_{n}>t_{0}\right)=1-F^{n}\left(t_{0}\right)$.We need to show that

$$
\int_{x}^{\infty} \overline{F_{(n)}}\left(t_{0}+y\right) d y \leq \overline{F_{(n)}}\left(t_{0}\right) \int_{x}^{\infty} \overline{F_{(n)}}(y) d y, \quad x \geq 0
$$

the inequality(4.1) obviously holds whenever $\overline{F_{(n)}}\left(t_{0}\right)=0$. For $\overline{F_{(n)}}\left(t_{0}\right) \neq 0$, the characterization result given by Definition(2.2-(ii)) may be reformulated as $: F \in N B U C-t_{0}$ iff

$$
\frac{F\left(t_{0}\right)}{1-F\left(t_{0}\right)} \int_{x+t_{0}}^{\infty} \bar{F}(u) d u . \leq \int_{x}^{x+t_{0}}[1-F(u)] d u
$$

the right-hand side of (4.2) satisfies

$$
\int_{x}^{x+t_{0}} \bar{F}(u) d u \leq \int_{x}^{x+t_{0}} \overline{F_{(n)}}(u) d u
$$

Also the left-hand side of (4.2) satisfies

$$
\int_{x+t_{0}}^{\infty} \frac{F\left(t_{0}\right)}{1-F\left(t_{0}\right)} \bar{F}(u) d u \geq \int_{x+t_{0}}^{\infty} \frac{F^{n}\left(t_{0}\right)}{1-F^{n}\left(t_{0}\right)}\left[1-F^{n}(u)\right] d u
$$

The last inequality(4.4) holds because 


$$
\begin{aligned}
& \int_{x+t_{0}}^{\infty}\left\{\frac{F\left(t_{0}\right)}{1-F\left(t_{0}\right)}[1-F(u)]-\frac{F^{n}\left(t_{0}\right)}{1-F^{n}\left(t_{0}\right)}\left[1-F^{n}(u)\right]\right\} d u \\
= & \int_{x+t_{0}}^{\infty} \frac{F\left(t_{0}\right)}{1-F\left(t_{0}\right)}[1-F(u)]\left\{1-F^{n-1}\left(t_{0}\right) \frac{\left[1-F\left(t_{0}\right)\right]\left[1-F^{n}(u)\right]}{\left[1-F^{n}\left(t_{0}\right)\right][1-F(u)]}\right\} d u \\
= & \int_{x+t_{0}}^{\infty} \frac{F\left(t_{0}\right)}{1-F\left(t_{0}\right)}[1-F(u)]\left\{1-F^{n-1}\left(t_{0}\right) \frac{\left[1+F(u)+\ldots+F^{n-1}(u)\right]}{\left[1+F\left(t_{0}\right)+\ldots+F^{n-1}\left(t_{0}\right)\right]}\right\} d u \\
\geq & \int_{x+t_{0}}^{\infty} \frac{F\left(t_{0}\right)}{1-F\left(t_{0}\right)}[1-F(u)]\left\{1-\frac{F^{n-1}\left(t_{0}\right)\left[1+F_{t_{0}}^{-1}+\ldots+F_{t_{0}}^{-(n-1)}\right]}{1+F\left(t_{0}\right)+\ldots+F^{n-1}\left(t_{0}\right)}\right\} d u \\
\geq & 0
\end{aligned}
$$

Since $F(u) \leq F^{-1}(u) \leq F_{t_{0}}^{-1}$ for $u \geq t_{0}$,we get

$$
\int_{x+t_{0}}^{\infty} \frac{F\left(t_{0}\right)}{1-F\left(t_{0}\right)}[1-F(u)] d u \geq \int_{x+t_{0}}^{\infty} \frac{F_{(n)}\left(t_{0}\right)}{\overline{F_{(n)}}\left(t_{0}\right)} \overline{F_{(n)}}(u) d u .
$$

From (4.3) and(4.5),we have

$$
\int_{x+t_{0}}^{\infty} \frac{F_{(n)}\left(t_{0}\right)}{\overline{F_{(n)}}\left(t_{0}\right)} \overline{F_{(n)}}(u) d u \leq \int_{x+t_{0}}^{\infty} \overline{F_{(n)}}(u) d u
$$

or

$$
\int_{x+t_{0}}^{\infty}\left[\frac{1}{\overline{F_{(n)}}\left(t_{0}\right)}-1\right] \overline{F_{(n)}}(u) d u \leq \int_{x+t_{0}}^{\infty} \overline{F_{(n)}}(u) d u
$$

or

$$
\int_{x+t_{0}}^{\infty} \frac{\overline{F_{(n)}}(u)}{\overline{F_{(n)}}\left(t_{0}\right)} d u \leq \int_{x}^{\infty} \overline{F_{(n)}}(u) d u
$$

i.e.

$$
\int_{x+t_{0}}^{\infty} \overline{F_{(n)}}(u) d u \leq \overline{F_{(n)}}\left(t_{0}\right) \int_{x}^{\infty} \overline{F_{(n)}}(u) d u
$$

Thus

$$
\int_{x+t_{0}}^{\infty} \overline{F_{(n)}}\left(t_{0}+y\right) d u \leq \overline{F_{(n)}}\left(t_{0}\right) \int_{x}^{\infty} \overline{F_{(n)}}(y) d y .
$$

This proves the reqired result. 


\subsection{Preservation of NBUC_ $\mathrm{t}_{0}$ class under Poisson Shock models.}

Suppose that a device is subjected to shocks occurring randomly in time according to a Poisson process with constant intensity $\lambda$. Suppose further that the device has probability $\bar{P}_{k}$ of surviving the first $K$ shocks, where

$1=\bar{P}_{0} \geq \bar{P}_{1} \geq \bar{P}_{2} \geq \ldots$. The survival function of the device is given by

$$
\bar{H}(t)=\sum_{k=0}^{\infty} e^{-\lambda t} \frac{(\lambda t)^{k}}{k !} \bar{P}_{k}
$$

For the discrete distribution $P_{k}$, it is well known that properties of $P_{k}$ are reflected in corresponding properties of the continuous life distribution $H(t)$. This is shown by Esary et al (1993) for IFR,IFRA,NBU and $N B U E$ classes by Klefsjö(1981), and Abouammoh and Ahmed (1988) for NBUFR. We will show that the same is true for the $N B U C-t_{0}$ class.

Definition(5.1).A discrete distribution and its survival probabilities

$$
\bar{P}_{k}=\sum_{j=k+1}^{\infty} P_{j}, K=0,1,2, \ldots \text { with finite mean } \mu=\sum_{k=0}^{\infty} \bar{P}_{k}
$$

are called discrete new better than used in convex ordering at $t_{0}$ (discrete $\left.N B U C-t_{0}\right)$ if

$$
\sum_{j=k}^{\infty} \bar{P}_{i+j} \leq \bar{P}_{i} \sum_{j=k}^{\infty} \bar{P}_{j}
$$

Theorem(5.2) The survival function $\bar{H}(t)$ in (5.1) is $N B U C-t_{0}$ if $\left\{\overline{P_{k}}\right\}_{k=0}^{\infty}$ has the discrete $N B U C-t_{0} \operatorname{property}(5.2)$.

Proof:

It must be shown that

$$
\int_{x}^{\infty} \bar{H}\left(u+t_{0}\right) d u \leq \bar{H}\left(t_{0}\right) \int_{x}^{\infty} \bar{H}(u) d u .
$$

Now consider,using (5.1) 


$$
\begin{aligned}
\int_{x}^{\infty} \bar{H}\left(u+t_{0}\right) d u & =\int_{x}^{\infty} \sum_{J=0}^{\infty} \frac{\left[\lambda\left(u+t_{0}\right]^{j}\right.}{j !} e^{-\lambda\left(u+t_{0}\right)} \overline{p_{j}} d u \\
& =\frac{1}{\lambda} e^{-\lambda t_{0}} \sum_{J=0}^{\infty} \overline{p_{j}} \sum_{m=0}^{j}\left(\begin{array}{c}
j \\
m
\end{array}\right) \frac{\left(\lambda t_{0}\right)^{j-m}}{j !} \int_{x}^{\infty}(\lambda u)^{j} e^{-\lambda u} d(u \lambda) . \\
& =\frac{1}{\lambda} e^{-\lambda\left(x+t_{0}\right)} \sum_{m=0}^{\infty} \sum_{j=m}^{\infty} \sum_{k=0}^{m} \overline{p_{j}} \frac{\left(\lambda t_{0}\right)^{j-m}}{(j-m) !} \frac{(\lambda x)^{k}}{k !}
\end{aligned}
$$

by integrating by parts $m$ times. we get

$$
\begin{aligned}
\int_{x}^{\infty} \bar{H}\left(u+t_{0}\right) d u & =\frac{1}{\lambda} e^{-\lambda\left(x+t_{0}\right)} \sum_{m=0}^{\infty} \sum_{j=m}^{\infty} \sum_{k=0}^{m} \overline{P_{j+m}} \frac{\left(\lambda t_{0}\right)^{j}}{(j) !} \frac{(\lambda x)^{k}}{k !} \\
& =\frac{1}{\lambda} \sum_{j=0}^{\infty} \frac{\left(\lambda t_{0}\right)^{j}}{(j) !} e^{-\lambda\left(t_{0}\right)} \cdot \sum_{k=0}^{\infty} \sum_{m=k}^{\infty} \overline{P_{j+m}} \frac{(\lambda x)^{k}}{k !} e^{-\lambda(x)} \\
& \leq \sum_{j=0}^{\infty} \frac{\left(\lambda t_{0}\right)^{j}}{(j) !} e^{-\lambda\left(t_{0}\right)} \cdot \bar{p}_{j} \cdot \sum_{k=0}^{\infty} \sum_{m=k}^{\infty} \bar{P}_{m} \cdot \bar{P}_{m} \frac{1}{\lambda} \frac{(\lambda x)^{k}}{k !} e^{-\lambda(x)}
\end{aligned}
$$

by using the $N B U C-t_{0}$ property

$$
\begin{aligned}
& =\sum_{j=0}^{\infty} \frac{\left(\lambda t_{0}\right)^{j}}{(j) !} e^{-\lambda\left(t_{0}\right)} \cdot \bar{p}_{j} \cdot \sum_{m=0}^{\infty} \bar{P}_{m} \cdot \sum_{k=0}^{m} \frac{(\lambda x)^{k}}{k !} \frac{1}{\lambda} e^{-\lambda(x)} \\
& =\bar{H}\left(t_{0}\right) \sum_{m=0}^{\infty} \bar{P}_{m} \int_{x}^{\infty} \frac{(\lambda u)^{m}}{m !} e^{-\lambda(u)} d u
\end{aligned}
$$

see Barlow and Proschan page 74,(5.4)

$$
\begin{aligned}
& =\bar{H}\left(t_{0}\right) \int_{x}^{\infty} \sum_{m=0}^{\infty} \frac{(\lambda u)^{m}}{m !} e^{-\lambda(u)} \bar{P}_{m} d u \\
& =\bar{H}\left(t_{0}\right) \int_{x}^{\infty} \bar{H}(u) d u .
\end{aligned}
$$

\section{REFERENCES}

Ahmad, I. A. (2001). Moments inequalities of aging families of distributions with hypothesis testing application. Journal of Statistical Planning and Inference, 92, 121-132.

Ahmad, I. A. and Mugdadi, A. R. (2004). Further moment inequalities of life distributions with hypothesis testing applications: The IFRA, NBUC, $D M R L$ classes. Journal of Statistical Planning and Inference, 120, 1-12..

A-Hameed, M. S. and Proschan, F. (1973). Nonstationary shock models. Stochastic Processes Applications, 1, 33-404.

A-Hameed, M. S. and Proschan, F. (1975). Shock models with underlying 
birth process. Journal of Applied Probability, 12, 18-28.

Alzaid, A., Kim, J. S. and Proschan, F. (1991). Laplace ordering and its applications. Journal of Applied Probability, 28, 116-130.

Barlow, R. E. and Proschan, F. (1981). Statistical Theory of Reliability and Life Testing. To Begin with. Silver Spring, M D.

Belzunce, F., Ortega, E. and Ruiz, J. M. (1999). The Laplace order and ordering of residual lives. Statistics \& Probability Letters, 42, 145-156.

Belzunce, F. (1999). On a characterization of right spread order by the increasing convex order. Statistics \& Probability Letters, 45, 103-110.

Block, H. W. and Savits, T. H. (1978). Shock models with NBUE survival. Journal of Applied Probability, 15, 621-628.

Chandra, N. K. and Roy, D. (2001). Some results on reversed failure rate. Probability in the Engineering and Informational Sciences, 15, 95-102.

Di Crescenzo, A. and Longobardi, M. (2002). Entropy-based measure of uncertainty in past lifetime distribution. Journal of Applied Probability, 39, 434-440.

Esary, J. D., Marshall, A. W. and Proschan, F. (1973). Shock models and wear processes. Annals of Probability, 1, 627-649.

Fagiuoli, E. and Pellerey, F. (1994). Mean residual life and increasing convex comparison of shock models. Statistics \& Probability Letters, 20, 337-345.

Hu, T., Kundu, A. and Nanda, A. K. (2003). A not on Bayesian imperfect repair model. Technical Report, Department of Statistics and Finance, University of Science and Technology of China.

Joag-Dev, K., Kochar, S. and Proschan, F. (1995). A general composition theorem and its applications to certain partial orderings of distributions. Statistics \& Probability Letters, 22, 111-119.

Karlin, S. (1968). Total Positivity. Vol I. Standford University Press, Stanford C A.

Lynch, J., Mimmack, G. and Proschan, F (1987). Uniform stochastic orderings and total positivity. Canadian Journal of Statistics, 15, 63-69.

Li, X. and Lu, J. (2003). Stochastic comparisons on residual life and inactivity time of series and parallel systems. Probability in the Engineering and Informational Sciences, 17, 267-75.

Lee, A. J. (1989). U-Statistics. Marcell-Dekker, New York, NY.

Mugdadi, A. R. and Ahmad, I.A. (2005). Moments inequalities derived from comparing life with its equilibrium form. Journal of Statistical Planning and 
Inference, to appear.

Nanda, A. K., Singh, H., Misra, N. and Paul, P.(2003). Reliability properties of reversed residual lifetime. Communications in Statistics- Theory \& Methods, 32, 2031-2041.

Pellerey, F. and Shaked, M. (1997). Characterizations of the IFR and DFR aging notions by means of the dispersive order. Statistics \& Probability Letters, 33, 389-393.

Pellerey, F.(1994). Shock models with underlying counting process. Journal of Applied Probability, 31, 156-166.

Perez-Ocon, R. and Gamiz-Perez, M. L. (1995). Conditions on the arrival process to obtain $H N B U E$ survival using a shock model. Communications in Statistics- Theory \& Methods, 24, 931-944.

Perez-Ocon, R. and Gamiz-Perez, M. L. (1996). HNBUE property in a shock model with cumulative damage threshold. Communications in Statistics- Theory \& Methods, 25, 345-360.

Ross, S. M. (1996). Stochastic Process. 2nd edition, John Wiley \& Sons, New York.

Ruiz, J. M. and Navarro, J. (1996). Characterizations based on conditional expectations of the double truncated distribution. Annals of the Institute of Statistical Mathematics, 48, 563-572.

Shaked, M. and Shanthikumar, J. G. (1994). Stochastic Orders and Their Applications. Academic Press, New York.

Shaked, M. and Wong, T. (1995). Preservation of stochastic orderings under random mapping by point processes. Probability in the Engineering and Informational Sciences, 9, 563-580.

Received: May 30, 2006 